\title{
Correction to: The Procedureless Elipse Gastric Balloon Program: Multicenter Experience in 1770 Consecutive Patients
}

\author{
R. lenca ${ }^{1} \cdot$ Mohammed Al Jarallah $^{2}$ - Adelardo Caballero ${ }^{3}$ - Cristiano Giardiello ${ }^{4}$ - Michele Rosa ${ }^{5}$. Sébastien Kolmer ${ }^{6}$. \\ Hugues Sebbag ${ }^{7}$. Julie Hansoulle ${ }^{8}$. Giovanni Quartararo ${ }^{9}$ - Sophie Al Samman Zouaghi ${ }^{10}$. Girish Juneja ${ }^{11}$. \\ Sébastien Murcia ${ }^{12} \cdot$ Roman Turro $^{13} \cdot$ Alberto Pagan $^{14} \cdot$ Faruq Badiuddin $^{15} \cdot$ Jérôme Dargent $^{16} \cdot$ Pierre Urbain $^{17}$. \\ Stefan Paveliu ${ }^{18} \cdot$ Rita Schiano di Cola ${ }^{4}$. Corrado Selvaggio ${ }^{5} \cdot$ Mohammed Al Kuwari $^{19}$
}

Published online: 31 August 2020

(C) The Author(s) 2020

\section{Correction to: Obesity Surgery (2020) 30:3354-3362. https://doi.org/10.1007/s11695-020-04539-8}

This article was originally published electronically on the publisher's internet portal on May 5, 2020 without open access. With the author(s)' decision to opt for Open Choice the copyright of the article changed on August 6,2020 to (C) The

The online version of the original article can be found at https://doi.org/10. 1007/s11695-020-04539-8

\section{R. Ienca}

roberta.ienca@gmail.com

Mohammed Al Jarallah

jarallah55@hotmail.com

Adelardo Caballero

info@institutodeobesidad.com

Cristiano Giardiello

cristiano.giardiello@pinetagrande.it

Michele Rosa

michelerosa@tiscali.it

Sébastien Kolmer

seb.kolmer@yahoo.fr

Hugues Sebbag

hsebbag.ppr@hotmail.fr

Julie Hansoulle

j.hansoulle@clarisclinic.com

Giovanni Quartararo

giovanni.quartararo@gmail.com

Sophie Al Samman Zouaghi dralsammanzouaghi@gmail.com

Girish Juneja

gijuneja@gmail.com
Author(s) 2020 and the article is forthwith distributed under a Creative Commons Attribution.

Open Access This article is licensed under a Creative Commons Attribution 4.0 International License, which permits use, sharing, adaptation, distribution and reproduction in any medium or format, as long as you give appropriate credit to the original author(s) and the source, provide a link to the Creative Commons licence, and indicate if changes were made. The images or other third party material in this article are included in the article's Creative Commons licence, unless indicated otherwise in a

\author{
Sébastien Murcia \\ sebastienmurcia@wanadoo.fr \\ Roman Turro \\ romanturro@gmail.com \\ Alberto Pagan \\ albertopagan@me.com \\ Faruq Badiuddin \\ faruq1@gmail.com \\ Jérôme Dargent \\ jerome.dargent@polyclinique-rillieux.fr \\ Pierre Urbain \\ urbain@chirobes.com \\ Stefan Paveliu \\ spaveliu2000@gmail.com \\ Rita Schiano di Cola \\ rita_schiano@libero.it \\ Corrado Selvaggio \\ selvaggio.corrado@virgilio.it \\ Mohammed Al Kuwari \\ drmalkuwari@gmail.com
}

Extended author information available on the last page of the article 
credit line to the material. If material is not included in the article's Creative Commons licence and your intended use is not permitted by statutory regulation or exceeds the permitted use, you will need to obtain permission directly from the copyright holder. To view a copy of this licence, visit http://creativecommons.org/licenses/by/4.0/.
Publisher's Note Springer Nature remains neutral with regard to jurisdictional claims in published maps and institutional affiliations.

\section{Affiliations}

\section{R. lenca ${ }^{1} \cdot$ Mohammed Al Jarallah $^{2} \cdot$ Adelardo Caballero $^{3} \cdot$ Cristiano Giardiello $^{4} \cdot$ Michele Rosa $^{5} \cdot$ Sébastien Kolmer $^{6}$. Hugues Sebbag ${ }^{7}$. Julie Hansoulle ${ }^{8}$. Giovanni Quartararo ${ }^{9}$. Sophie Al Samman Zouaghi ${ }^{10} \cdot$ Girish Juneja $^{11}$. Sébastien Murcia ${ }^{12} \cdot$ Roman Turro $^{13} \cdot$ Alberto Pagan $^{14} \cdot$ Faruq Badiuddin $^{15} \cdot$ Jérôme Dargent $^{16} \cdot$ Pierre Urbain $^{17}$. Stefan Paveliu ${ }^{18} \cdot$ Rita Schiano di Cola ${ }^{4}$. Corrado Selvaggio ${ }^{5} \cdot$ Mohammed Al Kuwari $^{19}$}

1 Weight Management Center, Nuova Villa Claudia Clinic, Rome, Italy

2 General Surgery Department, Jarallah German Clinic, Kuwait City, Kuwait

3 Bariatric Center, Instituto De Obesidad, Madrid, Spain

4 Emergency and Metabolic Surgery Department, Pineta Grande Hospital, Caserta, Italy

5 Nutritional Center, Micros Clinic, Modica, Italy

6 Digestive Surgery Department, Le Réseau Pondera, Mulhouse, France

7 Digestive Surgery Department, Polyclinique Du Parc Rambot, Aixen-Provence, France

8 Nutrional Center, Claris Clinic, Brussels, Belgium

9 General and Bariatric Surgery Unit, Villa Donatello, Florence, Italy

10 Digestive and Bariatric Surgery Department, Infirmerie Protestante, Caluire, France

11 Bariatric and Weight Loss Center, Cocoona Center, Dubai, UAE
12 Bariatric Surgery Department, Nouvelle Clinique Bordeaux Tondu, Floirac, France

13 Digestive Endoscopy Department, Centro Medico Teknon, Barcelona, Spain

14 Nutritional Center, Centro Integral Nutricion Baleares-Cinib, Palma de Mallorca, Spain

15 General and Obesity Surgery Department, BR Medical Suites, Dubai, UAE

16 Bariatric Surgery Department, Polyclinique Lyon Nord, Rillieux-laPape, France

17 General Surgery Department, Polyclinique Saint Privat, Boujansur-Libron, France

18 General and Obesity Surgery Department, Centre Medical Matisse, Nice, France

19 Bariatric Surgery Department, The Masters Medical Clinic, Doha, Qatar> 